\title{
Efectos de la capacitación y la retroalimentación sobre el plagio académico
} en estudiantes de Bachillerato

Effects of training and feedback on academic plagiarism in high school students

\author{
Diaz-Arce Dariel * \\ ddiaz@santana.edu.ec \\ Brito-González José Luis \\ jbrito@santana.edu.ec \\ Nieto-Trelles Valeria \\ Alexandra* \\ vnieto@santana.edu.ec \\ Muñoz-Arévalo Wilson \\ David* \\ wmunoza@santana.edu.ec
}

Unidad Educativa Santana*

ORCID:

https://orcid.org/0000-0002-

5952-9916*

https://orcid.org/0000-0002-

$8192-5026$

https://orcid.org/0000-0002-

7418-6691*

https://orcid.org/0000-0003-

1290-224X*

Para referenciar este artículo:

Diaz-Arce, D., Brito-González,

J.L. y Nieto-Trelles, A. y Muñoz-Arévalo, W.D. (2019). Efectos de la capacitación y la retroalimentación

sobre el plagio académico en estudiantes de

Bachillerato. Revista

ConCiencia EPG, 4(2),

24-41.

https://doi.org/10.3265 4/CONCIENCIAEPG.

4-2.3

\section{Resumen}

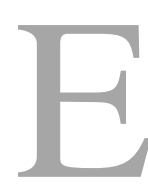

1 plagio académico es un problema que afecta a estudiantes de todos los niveles de enseñanza. El presente trabajo tuvo como objetivo evaluar la utilidad de un programa de capacitación y retroalimentación sobre la disminución del plagio académico en estudiantes de bachillerato. Se diseñó un estudio cuasiexperimental con una muestra de 61 estudiantes de bachillerato. Se establecieron sesiones de capacitación al inicio del año lectivo y posteriormente se recibieron durante el año cuatro trabajos académicos, cada uno de los cuales fue retroalimentado y evaluado para detectar diferentes formas de plagio, el nivel de gravedad y las mejorías observadas. Los resultados muestran una tendencia a reducir la frecuencia de formas graves de plagio hacia el final del estudio. El 44,3\% mostró signos claros de mejoría en la comprensión y aplicación de la citación y referenciado de fuentes y otro 29,5 $\%$ se mantuvo sin ninguna forma de plagio desde el inicio del curso. Hubo diferencias significativas en las mejorías observadas dependiendo del sexo y el rendimiento académico. Se concluye que la estrategia planteada puede ayudar a disminuir la frecuencia de plagio académico entre los estudiantes de bachillerato. Se recomiendas nuevos estudios experimentales en los que se puedan controlar más variables y observar la fortaleza de la propuesta.

Palabras clave: Plagio académico; fraude académico; ciberplagio; intervención educativa; deshonestidad académica. 
Abstract

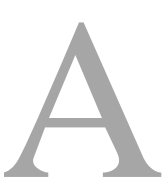

cademic plagiarism is a problem that affects students of all educational levels. The purpose of this work was to evaluate the effects of a training and feedback program on the reduction of academic plagiarism in high school students. A quasi-experimental design was used with a sample of 61 high school students. Training sessions were established at the beginning of the school year and subsequently four academic papers were received during the year, each of which was fed back and evaluated to detect different forms of plagiarism, the level of severity and the improvements observed. The results show a tendency to reduce the frequency of severe forms of plagiarism towards the end of the study. $44.3 \%$ showed clear signs of improvement in the understanding and application of the citation and reference of sources and another $29.5 \%$ remained without any form of plagiarism from the beginning of the course. There were significant differences in the improvements observed depending on sex and academic performance. It is concluded that the proposed strategy can help to reduce the frequency of academic plagiarism among high school students. New experimental studies are recommended in which more variables can be controlled and the strength of the proposal observed.

Keywords: Plagiarism; academic fraud, cyberplagiarism, educative intervention, academic dishonesty.

\section{Introducción}

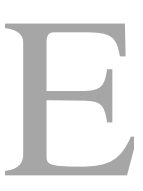

1 plagio académico es una forma de fraude, sea intencional o por descuido, y debe entenderse como un acto deshonesto mediante el cual una persona se apropia de las ideas de terceros para beneficio propio (Díaz Arce, 2015). La elevada frecuencia de este problema en todos los niveles de enseñanza (Alfaro Torres \& de Juan Juárez, 2014; Díaz Arce, 2016; Lai \& Weeks, 2009; SuredaNegre, Comas-Forgas \& Oliver-Trobat, 2015) y su significativo impacto en la formación ético-moral de los ciudadanos (Miranda Montecinos, 2013; RodríguezQuispe \& Sánchez-Baya, 2011), exige acciones urgentes para reducirlo.

Sobre este tema, en los últimos años se han desarrollado estudios enfocados fundamentalmente a la descripción e identificación de los factores condicionantes. Se presentan así diversas causales, algunas derivadas de las características de los estudiantes (procrastinación, bajo rendimiento académico, pocas habilidades y destrezas en redacción académica, entre otros), mientras otras se asocian a los docentes (estrategias didácticas poco motivadoras, no revisar los trabajos, no aplicar reglamentos, entre otros). Se destacan, además, otros factores intervinientes como el fácil y rápido acceso a la información y las bondades que ofrece la tecnología actualmente (Comas-Forgas \& Sureda-Negré, 2010; Mojeiko \& Rudkouski, 2019; Rebollo, Espiñeira \& Muñoz, 2017).

A pesar de que se tiene una idea más o menos clara de los posibles factores que propician la comisión de plagio académico entre los estudiantes, muy pocos son los estudios que se desarrollan para prevenirlo. En este caso se proponen estrategias enfocadas a elevar los procesos de detección, aplicación de reglamentos y sanción disciplinar, pero pocos dirigidos hacia su prevención (Gil-Cano, Novó-Rius \& PlanasCampistol, 2017; Sureda Negré et al., 2015).

Los autores del presente trabajo consideran que, si bien las estrategias punitivas podrían promover una disminución en la comisión de plagio entre los estudiantes, no son sostenibles cuando 
los condicionantes del comportamiento, la detección y el castigo, no están presentes. Por otro lado, si además de presentarse las posibles consecuencias de este problema en los reglamentos internos se fomenta una cultura hacia el respeto de la propiedad intelectual y el derecho de autor, podría lograrse un comportamiento ético más duradero y de impacto para la sociedad.

Una investigación previa en estudiantes de bachillerato demostró la utilidad del uso combinado de herramientas de detección de plagio (Turnitin) y la retroalimentación académica, sobre la disminución de este problema en los ensayos de la asignatura de Biología (Díaz Arce, 2015). A pesar de esto, Turnitin es una herramienta comercial que no está disponible para todos los educandos, a lo que se añade que sus análogas gratuitas no son estables en el tiempo (Díaz Arce, 2017).

Considerando lo anterior, se pretende responder la siguiente pregunta: ¿qué efectos poseen la retroalimentación y la capacitación sobre el plagio académico en estudiantes de bachillerato? Es un trabajo que busca sustentar el impacto del apoyo docente sobre la reducción de este problema, por lo que su metodología puede ser perfectamente replicable por otros autores en diferentes contextos educativos.

El plagio académico en los diferentes niveles de enseñanza.

La deshonestidad académica, y especialmente el plagio, es uno de los problemas más difundidos en todos los niveles educativos. En tal caso, se tienen reportes alarmantes en estudiantes desde educación secundaria básica y bachillerato hasta los profesionales que cursan determinados posgrados.

A nivel de la enseñanza media y media superior, los resultados de SuredaNegre et al. (2015) en las Islas Baleares indican que aproximadamente cuatro de cada cinco estudiantes han copiado fragmentos de internet en sus trabajos sin reconocer la fuente, mientras que tres de cada cuatro lo hicieron de fuentes impresas. Con esta elevada proporción también concuerda el estudio de Díaz Arce (2015) en Ecuador, en el que aproximadamente el $80 \%$ de los estudiantes de bachillerato mostró signos claros de plagio en ensayos académicos de la asignatura de Biología. Asimismo, otras investigaciones sustentan la hipótesis de que el plagio en la enseñanza media es un problema global, con una prevalencia que oscila entre $26 \%$ y $90 \%$ (Hassan \& Tunku Ahmad, 2017; Kukolja Taradi et al., 2010; Lai \& Weeks, 2009; Lee et al., 2016; Molina, Velásquez, Ríos, Calfucoy \& Cociña, 2011; Osaretin Ukpebaor \& Ogbebor, 2013; Santos et al., 2017; Sisti, 2007).

Por otro lado, a nivel universitario el panorama no es mejor. En este caso, resaltan en los trabajos realizados en España y las Islas Baleares, en los que se observa que aproximadamente dos de cada tres encuestados ha copiado o descargado fuentes de internet para entregarlo como propio sin reconocer la fuente. En esto último, aproximadamente el $80 \%$ sugiere que no cita las referencias consultadas en internet (Comas Forgas, Sureda Negre \& Oliver Trobat, 2011). Otras investigaciones en países europeos también sustentan una elevada frecuencia de este fenómeno entre los jóvenes universitarios (Pupovac, BilicZulle \& Petrovecki, 2008).

Asimismo, un estudio en Perú observó que alrededor de una de cada tres tesis de grado tenía signos de plagio en su introducción, de las cuales cerca de la mitad tenía copiado el $50 \%$ o más de sus párrafos (Saldaña-Gastulo, Quezada-Osoria, PeñaOscuvilca \& Mayta-Tristán, 2010). Por su parte, en Ecuador la prensa reportó que un $50 \%$ o más de las tesis de grado y posgrado 
del periodo de 2010 a 2013 tenían indicios de este problema (https://bit.ly/2Kvgg9p), lo que podría ser mucho mayor en trabajos entregados en las diferentes asignaturas (Cevallos Torres, Guijarro Rodríguez \& Domínguez Rivas, 2016).

Las causas del plagio académico.

De forma general, las investigaciones en estudiantes y docentes convergen en que el plagio académico es un problema multidimensional, con muchos factores potencialmente causales (Cebrián Robles et al., 2018; Diez-Martínez, 2015; Mojeiko \& Rudkouski, 2019; Ochoa \& Cueva, 2016; Rebollo, Epiñeira \& Muñoz, 2017). A pesar de esto, se pueden resumir en al menos tres dominios o grupos importantes: los que se deben a la actividad del docente, los que se deben a la características cognitivas y motivacionales de los estudiantes, y, por

Tabla 1

Principales causas del plagio académico separadas en tres dominios o grupos último, los que son externos al proceso de enseñanza aprendizaje (ver resumen en la Tabla 1).

Analizando a profundidad estas causas potenciales se podría plantear la hipótesis de que la causa del problema del plagio académico reside en los docentes, más que en los otros dos grupos. En tal caso, un docente motivador, con estrategias didácticas lúdicas o aplicativas a contextos cercanos de sus estudiantes, con una buena planificación y diversidad de las evaluaciones, así como capacitado para identificar, corregir y promover probidad académica, puede ayudar a los estudiantes a superar sus deficiencias en habilidades de redacción y competencias informacionales, así como a mejorar su interés por la asignatura, lo que redundaría en un mejor rendimiento académico.

\begin{tabular}{lll}
\hline $\begin{array}{l}\text { - Deficiente planificación } \\
\text { docente. }\end{array}$ & $\begin{array}{l}\text { - Diferencias de género, edad } \\
\text { y madurez psicológica. }\end{array}$ & $\begin{array}{l}\text { - Políticas y sanciones poco } \\
\text { claras o inexistentes. }\end{array}$ \\
- Poca motivación. & - Procrastinación. & $\begin{array}{l}\text { - Facilidades y comodidad de } \\
\text { la información en línea. }\end{array}$ \\
- Deficientes habilidades & -Pocas habilidades en el & - Bondades de las TIC. \\
para detectar, corregir y & manejo de la información y & - \\
promover cultura académica. & redacción académica. & - Comercialización de la \\
- Estrategias didácticas & - Bajo rendimiento. & educación.
\end{tabular}

- Falta de motivación.

Nota: Elaborado a partir de: Cebrián Robles et al., (2018); Diez-Martínez, (2015); Mojeiko \& Rudkouski (2019); Ochoa \& Cueva, (2016); Rebollo, Epiñeira \& Muñoz, (2017); Sureda Negre, Comas Forgas, \& Oliver Trobat, (2015).

Una de las limitantes fundamentales que se puede espetar a los estudios para identificar las causas potenciales del plagio académico, es la metodología empleada. En este caso, la mayoría de ellos no prosperan del análisis de las percepciones de docentes y estudiantes sobre este tema, con múltiples instrumentos o estrategias para recoger el dato primario. No cabe duda de la utilidad práctica de estos trabajos para aportar 
soluciones al problema y dirigir futuras acciones, pero no demuestran las posibles relaciones entre los factores analizados y el plagio en sí mismo. Tampoco se demuestra la relevancia e independencia de cada factor, para lo que podrían emplearse procedimientos estadísticos complejos como el análisis multivariado (Coscia Requena, 2017).

Dadas las limitantes anteriores, es necesario buscar otras estrategias sencillas que aporten evidencia de la importancia relativa de cada uno de estos factores, y que no se queden solamente en asociaciones estadísticas. En tal caso, si se parte de la hipótesis de que un factor A es una causa del problema B, si reducimos o eliminamos el primero, se debe al menos reducir el segundo. Este proceder no solo permite corroborar la relación entre las variables analizadas, sino que además aporta un detalle importante en la investigación de causalidad, que la causa antecede al efecto o consecuencia (Cortés, 2018).

Las propuestas para reducir el plagio académico.

Considerando las posibles causas, algunos autores sugieren estrategias para reducir el plagio académico. Tal es el caso de Gil-Cano et al., (2017) quienes realizan diez propuestas, la mayor parte de las cuales están relacionadas con el accionar del docente y de los centros de documentación de las instituciones escolares. Se resaltan, las que se enfocan a clarificar el concepto de plagio frente a la paráfrasis y los derechos de autor, el diseño y ejecución de sesiones de clase monotemáticas sobre el plagio, así como formar a los estudiantes para citar y referenciar correctamente.

Por otro lado, las intervenciones para reducir el plagio entre los alumnos son escasamente documentadas. Un estudio en estudiantes de bachillerato muestra que la retroalimentación y el acceso de los estudiantes a herramientas informáticas para detectar sus posibles infracciones, puede ayudar a reducir significativamente este problema (Díaz Arce, 2017).

El efecto positivo de emplear herramientas informáticas como Turnitin para prevenir el problema del plagio en estudiantes de diferentes niveles y edades, ha sido documentado por varios autores. Así se encuentran trabajos en Reino Unido (Ayo Ajelabi, 2013; Holi Ali, 2013), Botswana (Batana, 2010), Estados Unidos (Baker et al., 2008), Malasia y Australia (Cheah y Bretag, 2008) y Nueva Zelanda (Goddard y Rudzki, 2005). Sin embargo, esta herramienta comercial no está al alcance de todos los docentes y estudiantes y sus contrapartes gratuitas no son muy estables en el tiempo (Díaz Arce, 2017). Además, los estudios anteriores no demuestran el aprendizaje y las habilidades de los estudiantes para una redacción académica honesta, sin la presencia de esta herramienta.

Por ello, es importante el desarrollo de otras investigaciones en la que el uso de softwares "antiplagio" no sea el motivante o condicionantes fundamental de la conducta estudiantil para cumplimentar un código de honor respecto a los trabajos académicos. En esto pueden ser de utilidad las estrategias de acción tutorial propuestas por Ruiz Ledezma (2018), así como la retroalimentación adecuada propuesta por Díaz Arce (2015).

\section{Método}

Diseño: la investigación fue de tipo cuasiexperimental, en la que se evaluaron diferentes variables indicadoras de plagio académico en los estudiantes analizados antes y después de realizada la intervención de capacitación y retroalimentación. Cada estudiante tuvo su propio control, al observar sus resultados antes y después del cuarto trabajo evaluado. Ninguno de los estudiantes supo por anticipado que serían parte de esta 
investigación para evitar un posible efecto "placebo".

Como variable independiente se tuvo el tiempo o momento de medición (antes de la intervención vs. después de la intervención). Además, se consideró otras variables "variables intervinientes" como el sexo y el rendimiento académico que se resaltan en las fuentes consultadas como posibles factores que afectan este problema (Sureda Negre, Comas Forgas, \& Oliver Trobat, 2015).

Las variables dependientes fueron:

- Índice General de Similitud (IGS), el cual es aportado por la herramienta Turnitin e indica el porcentaje de similitud del documento analizado con otros documentos de Internet. Por ejemplo, un IGS $\geq 14 \%$ es solo indicadora de posible plagio por copy-paste, el mismo que debe corroborarse observando detenidamente los trabajos analizados. La revisión a fondo por parte del investigador además aporta información sobre otras formas de plagio académico no detectados directamente por este software (Díaz Arce, 2016). Se puede esperar una disminución en el IGS, a medida que la redacción académica sea más original, aunque esto depende de otros factores: una copia textual, bien citada, no tiene por qué considerarse plagio académico, a no ser que el trabajo indique poco aporte personal por Mashup como se indica a continuación.

- Nivel de gravedad del plagio: éste se define por una escala creciente de percepción de gravedad por los autores. Esta variable es importante para visualizar el posible cambio de la gravedad de la falta cometida al contrastar el antes y después del tratamiento experimental. Sus categorías o niveles se muestran a continuación:

5. Copia textual no citada o referenciada (Mayor gravedad).

4. Paráfrasis no citada o referenciada.

3. Poco aporte personal: Mashup o copias textuales bien citadas, pero abarcan más del $50 \%$ del cuerpo del trabajo.

2. Copia textual o casi textual mal citada (falta de comillas, inicio de comillas antes o después del inicio de la copia textual).

1. Sin plagio evidente.

Nivel de mejora: éste representa cuánto el estudiante mejoró o no su desempeño según el tipo de plagio detectado antes y después de la intervención. Para ello se aplicó la siguiente escala:

0: No mejora o empeora, cuando el nivel de plagio en ambos ensayos es menor o igual que el inicial.

1: Mejoría leve, cuando el estudiante presenta un trabajo final aún con plagio, pero con un nivel inferior de gravedad.

2: Mejoría alta, cuando el estudiante en su primer ensayo mostró plagio, pero en el último no.

3: Calidad superior, cuando el estudiante no hizo plagio 
en ninguno de los dos ensayos.

Participantes: la población de estudio estuvo conformada por todos los estudiantes de $1^{\circ}$ de bachillerato de la Unidad Educativa Santana. La muestra fue elegida por conveniencia, dada la accesibilidad a todos los datos requeridos para el estudio por parte de los participantes. En tal caso, fue de 61 estudiantes, lo que representó el 98,4\% de la población (un estudiante no estuvo presente en todas las capacitaciones y además se retiró de la institución antes de concluir el estudio); de éstos, 31 fueron mujeres y 30 hombres, con edades entre 15 y 16 años, y un rendimiento promedio en el primer parcial de 7,81/10. Los estudiantes estuvieron agrupados en tres paralelos con similar proporción por sexo y nivel académico según los datos del curso anterior. A pesar de lo anterior, en las pruebas diagnósticas realizadas al inicio del curso no hubo homogeneidad en sus resultados.

Procedimientos: todos los cursos fueron atendidos por un solo docente de la asignatura Biología, experimentado en redacción y publicación científica, así como en el manejo de la herramienta Turnitin. La estrategia de intervención se basó en las recomendaciones propuestas por Gil-Cano et al. (2017).

En un primer momento, todos los estudiantes recibieron al inicio de clases seis sesiones monotemáticas de $50 \mathrm{~min}$. de duración sobre el concepto de plagio académico, ¿cómo citar y referenciar documentos e imágenes de internet según las normas de la institución?, ¿cómo buscar y reconocer fuentes confiables en internet?, así como las características de la paráfrasis y las citas textuales. La información sobre este tema se resumió en videos y matrices de autoevaluación posteadas en el blog de la asignatura.
Posteriormente se diseñaron cuatro ensayos de investigación para todo el año lectivo, relacionados con los temas siguientes: Dimensiones celulares, Virus: ¿vivos o no?, Riesgos y beneficios del consumo de los ácidos grasos, Calentamiento global: ¿es la actividad humana una causal? Cada ensayo fue orientado en sus guías didácticas, en cuanto a extensión, criterios de evaluación y forma de entrega.

Cada uno de los ensayos tuvo una duración mínima de dos semanas desde su orientación, y se entregaron en formato Word. Después de su recepción, los documentos fueron subidos por el docente a la plataforma Turnitin para revisar su Índice General de Similitud (IGS) con otros trabajos de Internet. Ninguno de los estudiantes tuvo acceso a este programa durante el curso, pero sus trabajos fueron retroalimentados con los IGS y los párrafos de mayores problemas. Se les dio además la evaluación sobre la calidad de las fuentes empleadas, sus errores de citación y referenciado, así como los problemas de redacción, entre otros.

Como parte de las estrategias de retroalimentación, los estudiantes con signos de plagio evidente en su primer y segundo ensayos fueron entrevistados por uno de los docentes del área para identificar nuevamente las posibles causales que le llevaron a incumplir con sus obligaciones. A todos se les explicó una vez más las formas de prevenir el plagio en sus documentos y las consecuencias que podría tener el mismo, así como la posibilidad de mejorar el documento previamente enviado.

En todos los ensayos se computó el IGS y se revisaron a fondo para identificar posibles formas de plagio. En caso de detectarse algún problema de probidad académica, se clasificaron según la escala correspondiente al nivel de gravedad. El 
nivel de mejora se computó solamente en el ensayo inicial y el final.

Todos los datos fueron procesados en SPSS. v. 23 y los gráficos se realizaron con la ayuda del programa Microsoft Excel 2010. Se recogieron como posibles variables confusoras el rendimiento académico en lecciones del primer parcial del año, así como el sexo. Se describieron los resultados con los valores de la media aritmética y desviación estándar del IGS y del nivel de plagio, separados por sexo y rendimiento académico. Para comparar los datos pareados de los cuatro ensayos de cada estudiante, se empleó el test Friedman (más de dos grupos) y el test de Wilcoxon (dos grupos). Se emplearon estas pruebas no paramétricas porque los datos no siguen una distribución normal en alguno de los grupos analizados, lo que es un requisito para la aplicación de test estadísticos de tipo paramétrico. Posteriormente se analizaron las mejoras de los ensayos por gráficos de barras con las frecuencias relativas de presentación de cada categoría de esta variable. Asimismo, se utilizaron tablas de contingencia con análisis Chi-Cuadrado de independencia para analizar posible asociación entre el nivel de mejoras y las variables confusoras, sexo y rendimiento académico. Se consideró este análisis debido al posible impacto diferencial de estas variables en la presentación de plagio académico (Sureda Negre, Comas Forgas, \& Oliver Trobat, 2015) lo que nos ayudaría a esclarecer más el impacto de la estrategia planteada sobre estos estratos. Para estas comparaciones se emplearon el primer y último ensayo para realizar las comparaciones. Dos estudiantes no tenían el primer ensayo, por lo que en su lugar se presentó el resultado del segundo. En todos los casos el nivel de significancia empleado fue de $p \leq 0,05$.

\section{Resultados}

De forma general no se observó un cambio estadísticamente significativo en el IGS de los estudiantes en los cuatro ensayos realizados. No obstante, al segmentar la muestra según el sexo se observa una tendencia a aumentar el IGS en las mujeres desde el primero al último trabajo, lo que no se vio en los hombres. Asimismo, al comparar cada ensayo por sexo se detectaron diferencias significativas en esta variable para los ensayos 1, 2 y 4, siendo mayores los índices de similitud entre los hombres. Por otro lado, la similitud con trabajos de internet solo mostró diferencias en el primero de los ensayos realizados en el curso, siendo los estudiantes con calificaciones menores a $8 / 10$ puntos los que tienden a presentar un mayor porcentaje de similitud (Tabla 2).

En cuanto a los índices o niveles de plagio, a pesar de que se observa una tendencia general a disminuir la gravedad de las acciones durante el curso, esta reducción no fue estadísticamente significativa. Esta observación no se comportó de igual manera según el sexo, en el cual las mujeres no modificaron su índice de plagio, no así los hombres que disminuyeron el mismo desde un primer ensayo al último. Se debe notar además que en los tres primeros ensayos el nivel de plagio fue mayor entre los hombres, pero en el cuarto ensayo, no hubo diferencias significativas lo que sugiere una homogenización de la muestra hacia el final del año lectivo. Por otra parte, los estudiantes con un rendimiento inferior a $8 / 10$ puntos redujeron significativamente su nivel de plagio durante el curso, no así los de mayores calificaciones. A pesar se reduce la brecha o diferencia entre los niveles de plagio por ensayo según el rendimiento, aún en el cuarto ensayo se manifiesta un mayor índice de plagio entre los de menor desempeño académico en su primer parcial (Tabla 2). Estos resultados muestran en general que los estudiantes de menor rendimiento académico y los del sexo 
masculino, tienden a presentar mayores dificultades en cuanto a la prevención del plagio académico al inicio de la intervención, y además son aquellos que tienden a mejorar más durante el curso con las estrategias empleadas.

Tabla 2

IGS y nivel de gravedad del plagio en los diferentes ensayos, estratificado por sexo y rendimiento académico

\begin{tabular}{|c|c|c|c|c|c|c|c|}
\hline Variable & Estratos & Cat. & Ens. 1 & Ens. 2 & Ens. 3 & Ens. 4 & $p$ \\
\hline \multirow[t]{5}{*}{ IGS (\%) } & General & - & $26 \pm 17$ & $30 \pm 18$ & $25 \pm 15$ & $28 \pm 9$ & 0,118 \\
\hline & Sexo & $\mathrm{F}$ & $19 \pm 15$ & $25 \pm 13$ & $22 \pm 9$ & $25 \pm 7$ & 0,046 \\
\hline & & M & $32 \pm 18^{*}$ & $34 \pm 21^{*}$ & $28 \pm 19$ & $30 \pm 11^{*}$ & 0,364 \\
\hline & Rendimiento & $<8,0$ & $30 \pm 19^{*}$ & $30 \pm 18$ & $25 \pm 17$ & $28 \pm 11$ & 0,423 \\
\hline & & $\geq 8,0$ & $20 \pm 14$ & $29 \pm 18$ & $25 \pm 14$ & $27 \pm 6$ & 0,108 \\
\hline \multirow{5}{*}{$\begin{array}{l}\text { Nivel de } \\
\text { gravedad } \\
\text { del plagio }\end{array}$} & General & - & $2,5 \pm 1,7$ & $2,3 \pm 1,5$ & $2,2 \pm 1,5$ & $2,1 \pm 1,5$ & 0,191 \\
\hline & Sexo & $\mathrm{F}$ & $1,8 \pm 1,5$ & $1,8 \pm 1,2$ & $1,7 \pm 1,3$ & $2,1 \pm 1,6$ & 0,501 \\
\hline & & M & $3,2 \pm 1,7^{*}$ & $2,9 \pm 1,5^{*}$ & $2,8 \pm 1,6^{*}$ & $2,1 \pm 1,4$ & 0,028 \\
\hline & Rendimiento & $<8,0$ & $3,3 \pm 1,7$ & $2,7 \pm 1,6$ & $2,4 \pm 1,6$ & $2,4 \pm 1,6$ & 0,020 \\
\hline & & $\geq 8,0$ & $1,5 \pm 1,2 *$ & $1,9 \pm 1,2^{*}$ & $2,0 \pm 1,5$ & $1,8 \pm 1,3^{*}$ & 0,387 \\
\hline
\end{tabular}

Nota: IGS, Índice General de Similitud; Cat. Categoría de la variable; *, indica diferencias significativas entre las categorías de una misma variable en cada ensayo; $p$, probabilidad de error tipo I.

Al inicio del curso, el 54,1\% de los trabajos mostró alguno de los signos de plagio evaluados, mientras que al final, esta frecuencia se ubicó en 45,9 \%. La reducción más significativa fue en el plagio por copia textual no citada o referenciada, la que disminuye casi tres veces respecto al ensayo inicial $(\mathrm{p}=0,019)$ (Figura 1). Se debe indicar que estos resultados solo indican un leve aumento en la frecuencia de trabajos sin plagio académico, sin embargo, no toma en cuenta el total de estudiantes que mejoraron o no. 


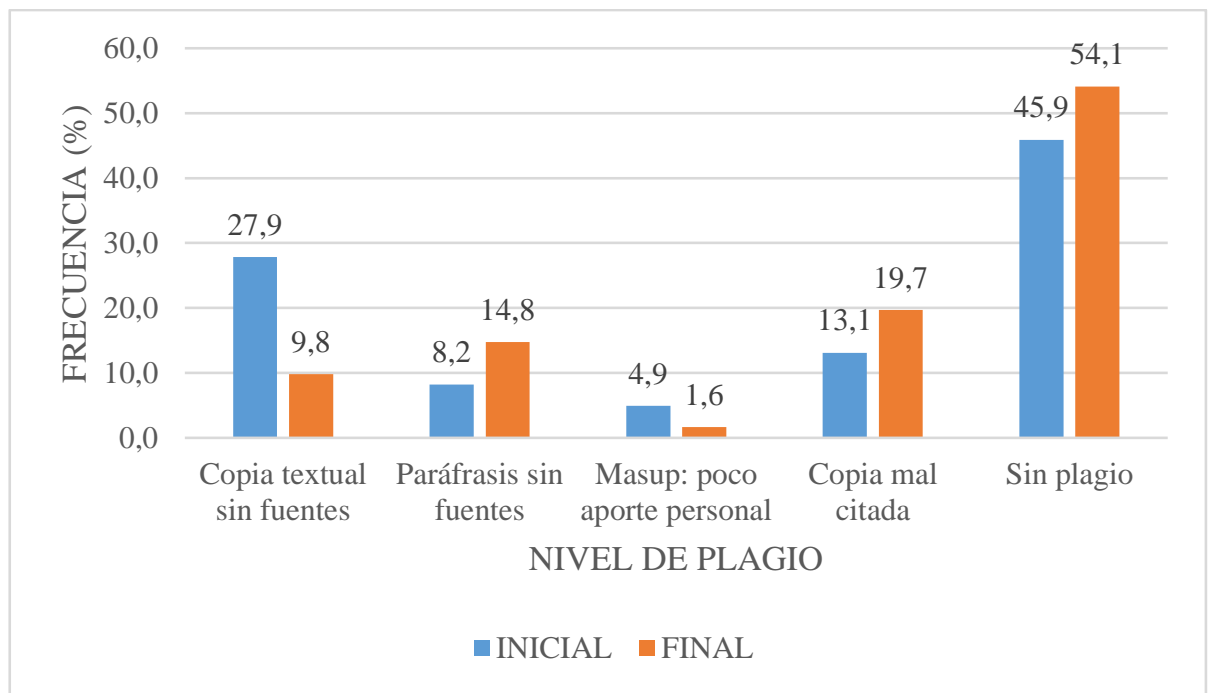

Figura 1. Frecuencia según tipo de plagio en los ensayos inicial y final.

Complementando los datos de la figura anterior, los resultados entre el primer y último ensayo (Figura 2), muestran que el $44,3 \%$ de los estudiantes tuvo signos de mejoría, de los cuales más de la mitad avanzó hasta no tener plagio académico. De los 33 estudiantes con algún nivel de plagio en su ensayo inicial, solo $4(12,1 \%)$ no mejoraron nada, $15(45,5 \%)$ pasaron a mostrar ensayos sin signos de este problema y $8(25,8 \%)$ pasaron a formas leves con ligeras dificultades en el no uso de comillas en las citas textuales, pero sí reconociendo la fuente de origen. Por su parte, también un $29,5 \%$ de la muestra se mantuvo sin plagio entre el primer y último trabajo entregado, lo que representa el $64,3 \%$ de los que inicialmente no tuvieron este problema.

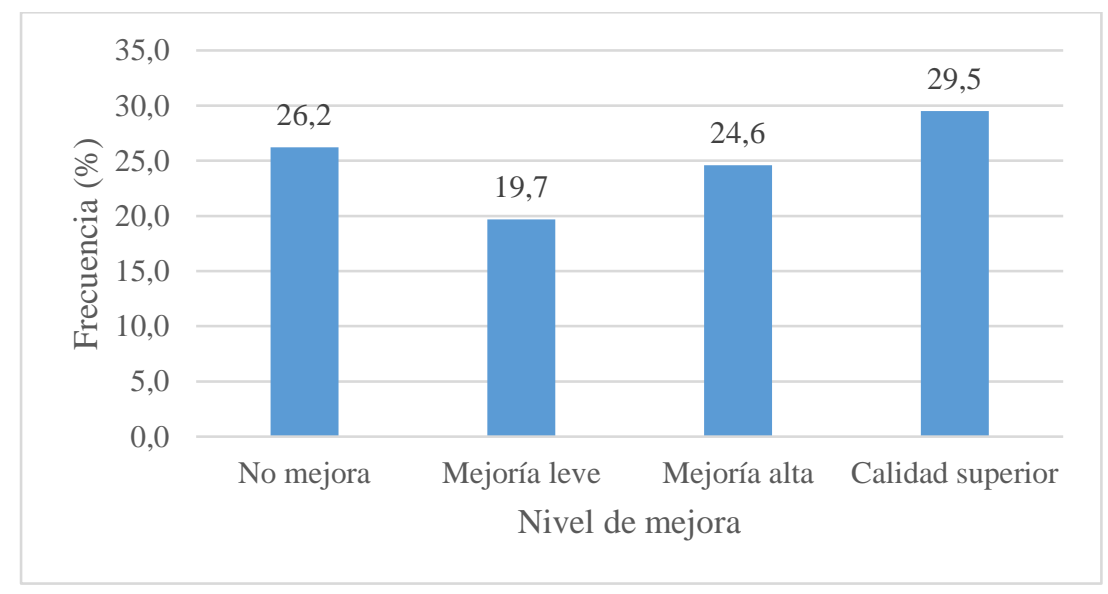

Figura 2. Niveles de mejora en la muestra de estudio.

Al separar el análisis por sexo se manifiestan diferencias significativas: los hombres tienden a presentarse más en las zonas de mejora y las mujeres más en la de trabajos de calidad superior. En el caso del rendimiento académico también se observan diferencias significativas, con los estudiantes de menor desempeño ubicándose más en las zonas de mejora, mientras que los que poseen mejores resultados académicos 
se presentan más en las zonas de calidad superior (Tabla 3). Estos resultados complementan los observados en la Tabla 2, respecto a las diferencias del IGS y nivel de gravedad del plagio observadas por sexo y

Tabla 3

Niveles de mejora según sexo y rendimiento académico de los estudiantes

\section{Resultado final $(n(\%))$}

\begin{tabular}{llccccc}
\cline { 3 - 6 } Variable & & $\begin{array}{c}\text { No mejora o } \\
\text { empeora }\end{array}$ & $\begin{array}{c}\text { Mejora } \\
\text { levemente }\end{array}$ & $\begin{array}{c}\text { Mejora } \\
\text { mucho }\end{array}$ & $\begin{array}{c}\text { Calidad } \\
\text { superior }\end{array}$ & $\mathrm{p}$ \\
\hline \multirow{2}{*}{ Sexo } & $\mathrm{F}$ & $8(25,8)$ & $4(12,9)$ & $5(16,1)$ & $14(45,2)$ & 0,036 \\
& $\mathrm{M}$ & $8(26,7)$ & $8(26,7)$ & $10(33,3)$ & $4(13,3)$ & \\
\hline \multirow{2}{*}{ Rendimiento } & $<8,0$ & $10(27,8)$ & $11(30,6)$ & $11(30,6)$ & $4(11,1)$ & 0,001 \\
& $\geq 8,0$ & $6(24,0)$ & $1(4,0)$ & $4(16,0)$ & $14(56,0)$ & \\
\hline Total & - & $16(26,2)$ & $12(19,7)$ & $15(24,6)$ & $18(29,5)$ & - \\
\hline
\end{tabular}

Nota: p, probabilidad de error tipo I.

\section{Discusión}

El plagio académico al inicio del curso afectó aproximadamente a uno de cada dos estudiantes. Esto contrasta con los reportes del $80 \%$ en estudiantes de tercero de bachillerato de la misma institución tres años antes (Díaz Arce, 2015). Estas diferencias podrían asociarse a las estrategias que se vienen realizando en el centro educativo durante este tiempo. No obstante, a nivel internacional el rango en que este problema afecta a los estudiantes de la enseñanza media es muy amplio, el cual va desde $26 \%$ a 90\% (Hassan \& Tunku Ahmad, 2017; Kukolja Taradi et al., 2010; Lai \& Weeks, 2009; Lee et al., 2016; Molina et al., 2011; Osaretin Ukpebaor \& Ogbebor, 2013; Santos et al., 2017; Sisti, 2007).

Otro de los elementos a destacar en el presente estudio es la significativa reducción de la forma más grave de plagio, la copia textual sin reconocer la fuente. Esto se debe a que se trata de la forma de plagio más fácil las calificaciones del primer parcial. De esta forma, se resalta más la importancia de un análisis por separado del impacto de la intervención en cada una de estas variables confusoras.

La posible influencia de la retroalimentación sobre la reducción del plagio académico en los estudiantes de bachillerato ya fue reportada previamente por Díaz Arce (2015). Trabajando con jóvenes de 17 a 18 años demostró que esta estrategia podría ser tan efectiva como para reducir la frecuencia de plagio por copypaste desde un $81 \%$ en un primer ensayo hasta un $20 \%$ en un tercero. No obstante, este trabajo empleó además una herramienta informática "antiplagio" a la que los estudiantes tenían acceso y podían revisar previamente sus manuscritos, para así pulirlos y reducir este problema. Esto es una limitante, sobre todo para aquellos estudiantes de otros lugares que no tengan acceso a este tipo de apoyo. Resultados similares se reportan en otros contextos y 
niveles educativos (Ayo Ajelabi, 2013; Davis \& Carroll, 2009).

En el presente estudio, si bien el docente utiliza una herramienta informática para detectar la posible copia de fuentes de internet, los estudiantes no poseen acceso a ella. Ello supone que la mejoría observada en los trabajos puede deberse en gran medida a las retroalimentaciones ofrecidas por el docente. Sin embargo, no puede descartarse que los estudiantes tengan cierta predisposición al saber que sus trabajos se están revisando con un software "antiplagio". Esto ha sido señalado ya con anterioridad por otro autor, quién empleando Turnitin sin aplicar retroalimentación logró reducir significativamente la frecuencia de trabajos con IGS $\geq 35 \%$ desde un $19,5 \%$ en un primer ensayo hasta $8,2 \%$ en un segundo (Batane, 2010). Las diferentes metodologías empleadas en ambos casos, limita la comparación, pero tales resultados sugieren que no debe descartarse la posibilidad de un accionar de los estudiantes, condicionado por el uso de esta herramienta.

A pesar de que las retroalimentaciones se realizaron a todos los ensayos de los alumnos, al parecer tienen un mayor efecto sobre los hombres y los de menor rendimiento académico.

La diferencia entre hombres y mujeres en cuanto a la incidencia de plagio es controversial. Si bien Honig \& Bedi (2012) y Molina et al. (2011) no encuentran diferencias respecto al sexo, otros autores sugieren que la copia textual está más extendida entre los hombres (Becker \& Ulstad, 2007; Mut-Amengual et al., 2012; Schlosser Montes, 2014; Sureda et al., 2015; Ummi Fa'iezah, 2010). Esto último concuerda con la observación realizada en el presente trabajo respecto a que los trabajos del sexo masculino presentan mayor IGS que el femenino. Además, es coherente con los estudios sobre posibles causas del plagio que ubican a los hombres como de mayor riesgo a cometer esta infracción (Dias \& Bustos, 2014; Gómez, Salazar \& Vargas, 2013; Sureda et al., 2015).

Por otro lado, el bajo rendimiento académico ha sido señalado por varios autores como una posible causa de la comisión de plagio. Es probable que la presión de obtener mejores calificaciones para promover una asignatura sea la motivación fundamental de este problema (Calvert \& Moorman 2012; Mojeiko \& Rudkouski, 2019; Ma et al., 2008; Ramos Quispe et al., 2019). En este sentido MoreyLópez et al. (2013) muestran que entre estudiantes de secundaria obligatoria que repitieron cursos, la frecuencia de plagio es mayor, especialmente de fuentes de internet. Detectaron también correlaciones negativas respecto a la frecuencia de plagio y el rendimiento académico, en diferentes asignaturas, entre las que se encuentra Ciencias de la Naturaleza. Tales resultados son concordantes con los observados en la presente investigación.

De forma similar, entre las posibles causas del plagio académico se citan por varios autores la falta de habilidades para citar y referenciar, así como la falta de acompañamiento docente en el proceso de escritura académica libre de fraude (Calvert y Moorman, 2012; Cebrián Robles et al., 2018; Ochoa \& Cueva, 2016; Rebollo et al., 2017). En el presente estudio la retroalimentación se enfocó entre otros detalles a la mejora de estos aspectos, especialmente a corregir los problemas de citación-referenciado de las copias textuales y las paráfrasis. Esto se demuestra en la significativa reducción del nivel de plagio entre los estudiantes hombres, sin disminuir mucho el IGS, así como el poco cambio en esta variable en las mujeres a pesar de que su IGS aumenta.

Hasta el momento, los estudios consultados sobre posibles intervenciones para reducir esta forma de deshonestidad 
académica no analizan las variables sexo y rendimiento académico de los estudiantes. Es por ello, que los resultados presentados en este trabajo son alentadores al respecto, ya que sugieren que un alumno puede mejorar su probidad académica aun teniendo bajo desempeño académico en una asignatura. También sugieren que, si bien el sexo masculino puede ser un factor de riesgo para el plagio, también se puede influir sobre ellos con retroalimentaciones oportunas para reducir este tipo de fraude.

El diseño del estudio plantea como limitación importante que no se dispuso de un grupo de contraste sobre el cual se aplicara otra estrategia de corrección de plagio académico, como lo es mediante el uso de Turnitin. Esto obedece fundamentalmente a que los estudiantes del primer año de bachillerato no tenían acceso a este recurso, el mismo que se ofrece en la institución para los años subsecuentes. Esta herramienta demostró su efectividad en estudiantes de 3er curso de Bachillerato, en los que ayudó a reducir el plagio por copypaste de un $81 \%$ en un ensayo inicial de Biología hasta un $20 \%$ en el tercero. A pesar de esto, solo se observó mejoría en la citación-parafraseado-referenciado en un $36 \%$ de los participantes (Díaz Arce, 2015). La presente investigación, salvando las diferencias temporales y de edad de los

\section{Referencias}

Alfaro Torres, P., \& de Juan Juárez, T. (2014). El plagio académico: formar en competencias y buenas prácticas universitarias. RUIDERAe: Revista de Unidades de Información, 6, 1-20 https://revista.uclm.es/index.php/ruid erae/article/view/637

Ayo Ajelabi, P. (2013). Assessment of application of an online academic participantes del trabajo citado muestra que, siguiendo otra estrategia de retroalimentación y la capacitación pertinente al inicio del curso, puede ayudar a que un $44,3 \%$ de los participantes mejore en este aspecto, mientras que otro $29,5 \%$ se mantenga sin cometer este tipo de fraude. Lo anterior constituye una evidencia de la utilidad práctica de la propuesta realizada.

\section{Conclusiones}

El plagio en los ensayos académicos es frecuente entre los estudiantes de primero de bachillerato de la institución analizada, especialmente entre los de menor rendimiento y los del sexo masculino. La gravedad de este problema se reduce al emplear una secuencia inicial de capacitación y la posterior retroalimentación oportuna de los trabajos, lo que podría transformarse en una estrategia adecuada para combatir esta infracción en las diferentes instituciones a través de la corrección de los problemas de citaciónreferenciado. Se deben realizar más estudios en los que se evalúe directamente la fortaleza de la estrategia sugerida frente a otras formas empleadas en las instituciones educativas para prevenir este problema. Para ello podrían emplearse otros tipos de estudios más controlados y que limiten el posible impacto de variable confusoras como el sexo y el rendimiento académico. plagiarism detector for british teacher trainees. Journal of Educational and Instructional Studies In The World, 3(2), 135-142.

Baker, R.K., Thornton, B., y Adams, M. (2008). An evaluation of the effectiveness of Turnitin.Com as a tool for reducing plagiarism in graduate student term papers. College 
Teaching Methods \& Styles Journal, 4(9), 1-4. https://clutejournals.com/index.php/C TMS/article/download/5564/5647

Batane, T. (2010). Turning to Turnitin to Fight Plagiarism among University Students. Educational Technology \& Society, 13(2), 1-12. https://www.researchgate.net/profile/ Tshepo_Batane/publication/2203740 40_Turning_to_Turnitin_to_Fight_Pl agiarism_among_University_Student s/links/54ec51700cf2465f532df587/T urning-to-Turnitin-to-FightPlagiarism-among-UniversityStudents.pdf\#page $=6$

Becker, D. A. y Ulstad, I. (2007). Gender Differences in Student Ethics: Are Females Really More Ethical? Plagiary: Cross-Disciplinary Studies in Plagiarism, Fabrication, and Falsification, 77-91. https://quod.lib.umich.edu/p/plag/524 0451.0002.009/--gender-differencesin-student-ethics-are-females-reallymore?rgn=main;view=fulltext

Calvert Evering, L., \& Moorman, G. (2012). Rethinking plagiarism in the digital age. Journal of Adolescent \& Adult Literacy, 56(1), 35-44. https://doi.org/10.1002/JAAL.00100

Cebrián-Robles, V., Raposo-Rivas, M., Cebrián-de-la-Serna, M. y SarmientoCampos, J.A. (2018). Percepción sobre el plagio académico de estudiantes universitarios españoles. Educación XX1, 21(2), 105-129. https://doi.org/10.5944/educXX1.200 62

Cevallos Torres, L., Guijarro Rodríguez, A., \& Domínguez Rivas, L. L. (2017). Factores que inciden en el mal uso de la información en trabajos de investigación científica. Revista
Didasc@lia: Didáctica y Educación, $\operatorname{VII}(4)$, 57-74. http://refcale.uleam.edu.ec/index.php /didascalia/article/viewFile/1503/848

Cheah, S.W. y Bretag, T. (2008). Making technology work for academic integrity in Malaysia, en: $3 r d$ International Plagiarism Conference Refereed Proceedings, Newcastle-onTyne, U.K. 23-25. http://www.plagiarismadvice.org/rese arch-papers/item/makingtechnologywork-for-academicintegrity-in-malaysia

Comas Forgas, R., Sureda Negre, J., Trobat, M. O. (2011). Prácticas de citación y plagio académico en la elaboración textual del alumnado universitario. Teoría de la Educación. Educación y Cultura en la Sociedad de la Información, 12(1), 359-385. https://www.redalyc.org/pdf/2010/20 1021400017.pdf

Comas-Forgas, R., \& Sureda-Negre, J. (2010). Academic plagiarism: Explanatory factors from students' perspective. Journal of Academic Ethics, 8(3), 217-232. https://doi.org/10.1007/s10805-0109121-0

Cortés, F. (2018). Observación, causalidad y explicación causal. Perfiles latinoamericanos, 26(52). https://dx.doi.org/10.18504/p12652001-2018

Coscia Requena, C. (2017). Métodos estadísticos para evaluar la causalidad en estudios observacionales. (Tesis de Maestría). Universidad Complutense de Madrid, España. https://eprints.ucm.es/43964/1/TFM_ ClaudiaCosciaRequena.pdf 
Davis, M. y Carroll, J. (2009). Formative feedback within plagiarism education: Is there a role for text-matching software? International Journal for Educational Integrity, 5(2), 58-70. https://ojs.unisa.edu.au/index.php/IJE I/article/download/614/471

Dias, P. C., \& Bastos, A. S. (2014). Plagiarism in Portugal-secondary education teachers' perceptions. Procedia-Social and Behavioral Sciences, 116, 2598-2602. https://doi.org/10.1016/j.sbspro.2014. 01.618 .

Díaz Arce, D. (2015). El uso de Turnitin con retroalimentación mejora la probidad académica de estudiantes de bachillerato. Ciencia, Docencia y Tecnología, 26(51), 197-216. http://www.redalyc.org/articulo.oa?id $=14542676009$

Díaz Arce, D. (2016). Plagio académico en estudiantes de bachillerato: ¿qué detecta Turnitin? RUIDERAe: Revista de Unidades de Información. 9, 31. https://revista.uclm.es/index.php/ruid erae/article/view/1146

Díaz Arce, D. (2017). Evaluación del desempeño de tres herramientas antiplagio gratuitas en la detección de diferentes formas de copy-paste procedentes de Internet. Edutec. Revista Electrónica De Tecnología Educativa, (59), a354. https://doi.org/10.21556/edutec.2017. 59.812

Diez-Martínez, E. (2015). Deshonestidad académica de alumnos y profesores: Su contribución en la desvinculación moral y corrupción social. Sinéctica, (44), 1-17. http://www.scielo.org.mx/scielo.php? $\mathrm{pid}=\mathrm{S} 1665$ -
109X2015000100014\&script=sci_art text

Gil-Cano, D., Nonó Rius, B., \& PlanasCampistol, I. (2017). Diez propuestas para evitar el plagio entre los estudiantes universitarios. BiD, (39). http://bid.ub.edu/pdf/39/es/gil.pdf

Goddard, R. y Rudzki, R. (2005). Using an electronic text-matching tool (Turnitin) to detect plagiarism in a New Zealand university. Journal of University Teaching \& Learning Practice, 2(3), 58-63. https://files.eric.ed.gov/fulltext/EJ105 9505.pdf

Gómez, J., Salazar, I., \& Vargas, P. (2013). Dishonest behavior and plagiarism by university students: An application to management studies. Procedia-Social and Behavioral Sciences, 83, 766770 .

https://doi.org/10.1016/j.sbspro.2013. 06.144 .

Hassan, R., \& Tunku Ahmad, T. B. (2017). Internet Plagiarism among Form Four Chemistry Students of a Selected Malaysian Secondary School: Exploring Its Prevalence, Correlates and Gender Differences. IIUM Journal of Educational Studies, 5(2), 54-72.

https://doi.org/10.31436/ijes.v5i2.206

Holi Ali, H.I. (2013). Minimizing cyberplagiarism through Turnitin: faculty's \& students' perspectives. International Journal of Applied Linguistics \& English Literature, 2(2), 33-42. https://doi.org/10.7575/aiac.ijalel.v.2 n. 2 p. 33

Honig, B. y Bedi, A. (2012). The Fox in the Hen House: A critical examination of plagiarism among members of the 
Academy of Management. Academy of Management Learning \& Education, 11(1): 101-123. https://doi.org/10.5465/amle.2010.00 84

Kukolja Taradi, S., Taradi, M., Knežević, T., \& Đogaš, Z. (2010). Students come to medical schools prepared to cheat: a multi-campus investigation. Journal of Medical Ethics, 36(11), 666-670. https://doi.org/10.1136/jme.2010.035 410.

Lai, K. W., \& Weeks, J. J. (2009). High school students' understanding of eplagiarism: some New Zealand observations. CINZS: LTT, 21(1), 115.

https://www.otago.ac.nz/cdelt/otago0 67253.pdf

Lee, C. W. Y., Chu, S. K. W., Cheng, J. O. Y., \& Reynolds, R. (2016, October). Plagiarism-free inquiry project-based learning with UPCC pedagogy. In Proceedings of the 79th ASIS\&T Annual Meeting: Creating Knowledge, Enhancing Lives through Information \& Technology (p. 33). American Society for Information Science.

https://onlinelibrary.wiley.com/doi/p df/10.1002/pra2.2016.14505301033

Ma, H. J., Wan, G., \& Lu, E. Y. (2008). Digital cheating and plagiarism in schools. Theory Into Practice, 47(3), 197-203.

https://doi.org/10.1080/00405840802 153809.

Miranda Montecinos, A. (2013). Plagio y ética de la investigación científica. Revista chilena de derecho, 40(2), 711-726. https://dx.doi.org/10.4067/S071834372013000200016
Mojeiko, V., \& Rudkouski, P. (2019). Plagiarism among Belarusian students: Contributory factors, consequences, and solutions. Ostrogorski Centre, Minsk. https://belarusdigest.com/wpcontent/uploads/2019/04/RudkouskiMojeiko-Plagiarism-Paper.pdf

Molina, F., Velásquez, J. D., Ríos, S., Calfucoy, P. A., \& Cocina, M. (2011). El fenómeno del plagio en documentos digitales: un análisis de la situación actual en el sistema educacional chileno. Revista Ingeniería de Sistemas, XXV, 5-28. http://www.dii.uchile.cl/ ris/RISXX V/plagio.pdf

Morey-López, M., Sureda-Negre, J., OliverTrobat, M. F., \& Lluc-Comas-Forgas, R. (2013). Plagio y rendimiento académico entre el alumnado de Educación Secundaria Obligatoria. Estudios sobre Educación, 24,225244.

https://dadun.unav.edu/bitstream/101 71/29571/2/MOREY.pdf

Mut-Amengual, B., Morey-López, M., Comas-Forgas, R., y Sureda-Negre. J. (2012). Prevalencia en la comisión de ciberplagio entre alumnado de eso atendiendo a los resultados académicos en asignaturas del área de lengua y literatura. EDUTEC. Revista Electrónica de Tecnología Educativa; 40.

http://edutec.rediris.es/Revelec2/Rev elec40/pdf/Edutec-

e_n40_Mut_Morey_Comas_Sureda.p $\mathrm{df}$

Ochoa, L., \& Cueva, A. (2016). Percepciones de estudiantes acerca del plagio: datos cualitativos. Encuentros, Universidad Autónoma del Caribe, 14(2), 25-41. 
http://dx.doi.org/10.15665/re.v14i2.8 22

Osaretin Ukpebor, C., \& Ogbebor, A. (2013). Internet and plagiarism: Awareness, attitude and perception of students of secondary schools. International Research: Journal of Library and Information Science, 3(2), 254-267. https://irjlis.com/internet-plagiarismawareness/

Pupovac, V., Bilic-Zulle, L., Petrovecki, M. (2008). "On academic plagiarism in Europe. An analytic approach based on four studies". In: R. COMAS, J. SUREDA (coords.). "Academic cyberplagiarism" [online dossier]. Digithum. No. 10. UOC. http://www.uoc.edu/digithum/10/dt/e ng/pupovac_bilic-

zulle_petrovecki.pdf

Ramos Quispe, T., Núñez, D., Froilan, E., Arias, I., Gerardo, M., Arias Chávez, D., \& Caurcel Cara, M. J. (2019). Actitudes hacia el plagio en estudiantes de Administración de Empresas de dos universidades privadas en Arequipa. Propósitos y Representaciones, 7(1), 33-45. http://dx.doi.org/10.20511/pyr2019.v $7 \mathrm{n} 1.264$

Rebollo, N., Espiñeira, E. M., \& Muñoz, J. M. (2017). Atribuciones causales en el plagio académico por parte de los estudiantes universitarios. Revista de Estudios e Investigación en Psicología y Educación, Extr. (6), 192-196.

https://doi.org/10.17979/reipe.2017.0 .06 .2453

Rodríguez-Quispe, N., \& Sanchez-Baya, M. (2011). Plagio, una falta de Ética. Revista Científica Ciencia Médica, 14(2), 6-7. http://www.scielo.org.bo/scielo.php? script=sci_arttext\&pid=S1817$74332011000200002 \& \operatorname{lng}=$ es\&tlng= es.

Ruiz Ledesma, E. F. (2018). Plan de acción tutorial para evitar el plagio en materias de formación básica. RIDE. Revista Iberoamericana para la Investigación y el Desarrollo Educativo, 8(16), 464-492. https://doi.org/10.23913/ride.v8i16.3 52.

Saldaña-Gastulo, J. J., Quezada-Osoria, C. C., Peña-Oscuvilca, A., \& MaytaTristán, P. (2010). Alta frecuencia de plagio en tesis de medicina de una universidad pública peruana. Revista peruana de medicina experimental y salud pública, 27(1), 63-67. http://www.scielo.org.pe/pdf/rins/v27 n1/a11v27n1.pdf

Santos, C. C., Santos, P. S., Santana, M. C., Masuda, H., Barboza, M. B., \& Vasconcelos, S. M. (2017). Going beyond academic integrity might broaden our understanding of plagiarism in science education: A perspective from a study in Brazil. Anais da Academia Brasileira de Ciências, $\quad$ 89(1), 757-771. http://dx.doi.org/10.1590/00013765201720160474

Schlosser Montes, K. (2014). La percepción del plagio académico de los estudiantes y docentes de las facultades de arquitectura, derecho e ingeniería en la Universidad Rafael Landívar (Tesis de Maestría). Universidad Rafael Landívar, Facultad de Humanidades, Guatemala. http://biblio3.url.edu.gt/Tesario/2014/ 05/83/Schlosser-Karen.pdf 
Sisti, D.A. (2007). How Do High School Students Justify Internet Plagiarism? ETHICS \& BEHAVIOR, 17(3), 215231 , https://doi.org/10.1080/10508420701 519163.

Sureda Negre, J., Comas Forgas, R. L., \& Oliver Trobat, M. F. (2015). Plagio académico entre alumnado de secundaria y bachillerato: Diferencias en cuanto al género y la procrastinación. Comunicar, (44),
103-111.

http://dx.doi.org/10.3916/C44-201511

Ummi Fa'iezah, L. (2010). Gender differences in plagiarism attitudes among indonesian university students in Perth Australia. Journal of Education, 1(2), 1-18.

https://journal.uny.ac.id/index.php/jo e/article/download/191/136 